\title{
Gestión integral y desarrollo deportivo en la región de
}

San Martín, 2020

\author{
Paolo Patricio Philipps Arévalo \\ Philipps2011@ hotmail.com
}

\author{
Dr. Keller Sánchez Dávila \\ ksanchezd@ucv.edu.pe
}

Universidad César Vallejo

Tarapoto-Perú

\section{RESUMEN}

La investigación tuvo como objetivo diseñar una propuesta de gestión integral que contribuya al desarrollo deportivo en la región de San Martín, 2020. El estudio es de tipo básico y diseño descriptivo propositivo, se aplicó una encuesta con la finalidad de recolectar la información brindada por los participantes, después de la recolección de los datos brindada por los participantes, estas fueron digitalizadas y procesadas mediante software estadístico. En relación a la gestión integral, mediante las investigaciones realizadas queda demostrado que es un componente fundamental para el máximo aprovechamiento las capacidades y recursos institucionales en pro del desarrollo y crecimiento empresarial e institucional, percibir de igual manera su deterioro, de la misma forma en el momento en que ha terminado su periodo de vida para sanear los recursos productivos con los que efectivamente cuentan las instituciones, de esta manera se pueda aplicar otras medidas, sin dejar de lado la gestión integral. En cuanto al desarrollo deportivo, en base a los investigaciones analizadas y estudiadas, se concluye que, es una abstracción para crear personas competitivas, íntegras y sobresalientes, porque los deportes se pueden hacer como ejercicios, educación, entretenimiento, recreación, logro, profesión, política, negocios, industria y varios otros aspectos de cultura humana. También, se demostró cómo a través de un proceso evolutivo en el tiempo, el deporte ha llegado a afectar positivamente a otros contextos, incluyendo ciertamente la educación y la escuela, la industria del turismo y lo concerniente cuestiones socioeconómicas.

Palabras clave: gestión integral; desarrollo deportivo; alto rendimiento. 


\title{
Comprehensive management and sports development in the Sint Maarten region, 2020
}

\begin{abstract}
The research aimed to design a comprehensive management proposal that contributes to sports development in the San Martín region, 2020. The study is of basic type and descriptive design propositional, a survey was applied with the purpose of collecting the information provided by the participants, after the collection of the data provided by the participants, were digitized and processed using statistical software. With regard to integrated management, research has shown that it is a key component for maximizing the use of institutional capacities and resources for enterprise and institutional development and growth, In the same way, we can perceive its deterioration, in the same way at the moment when it has finished its period of life in order to clean up the productive resources that the institutions actually have, in this way we can apply other measures, without neglecting integral management. As for sports development, based on the researchers analyzed and studied, it is concluded that, is an abstraction to create competitive, upright and outstanding people, because sports can be done as exercises, education, entertainment, recreation, achievement, profession, politics, business, industry and various other aspects of human culture. It was also shown how through an evolutionary process over time, sport has come to positively affect other contexts, certainly including education and school, the tourism industry and socio-economic issues.
\end{abstract}

Keywords: comprehensive management; sports development; high performance.

Artículo recibido: 20. Julio. 2021 Aceptado para publicación: 18. Agosto. 2021 Correspondencia: Philipps2011@ @otmail.com Conflictos de Interés: Ninguna que declarar 


\section{INTRODUCCIÓN}

En el pasado, Estados Unidos tuvo problemas en dar satisfacción a las necesidades de alto rendimiento de sus deportistas con respecto a las competiciones internacionales, por lo que, mediante una gestión integral, el Comité Olímpico de Estados Unidos desarrolló tres Centros de Entrenamiento Olímpico (OTC) estratégicamente distribuidos en todo el Estado para mejorar la competitividad deportiva. De acuerdo con Moreau y Nabhan (2012) estos OTC se hallan localizados de forma diagonal en los Estados Unidos, de noreste hasta el sudoeste y han sido creados para ofertar un espectro extenso de servicios de alto rendimiento con un eje regional. De esta manera, los Estados Unidos gestiona integralmente a través de la federación de deportes relacionada o NGB que elige a sus competidores, para prepararlos en un OTC. Estos ofrecen una amplia gama de ayuda a los competidores, como cena, alojamiento, preparación de destinos, lugares deportivos, transporte cercano, administraciones para competidores y programas de avance de expertos. Normalmente, las administraciones de ejecución atlética que se ofrecen en los OTC también incluyen medicamentos para juegos, fisiología de la práctica, perseverancia y bienestar, nutrición deportiva, investigación del cerebro deportivo e investigación biomecánica.

Según el Gobierno de Colombia (2018), en cuanto al deporte y la diversión para el mejoramiento vital de las personas, para la conjunción y el apego social, solo el $51 \%$ de la población en algún lugar en el rango de 18 y 64 años realiza ensayos de trabajo activo, ubicándose en el puesto 23 del ranking mundial, a pesar de ello solo 35 de cada 100 escenarios deportivos del país se encuentran en buen estado, 46 en regular estado y 16 en mal estado. Por lo que muchos deportistas desisten en practicar algún deporte y pasan a tener un estilo de vida sedentario poniendo en riesgo su salud frente a riesgos de enfermedades cardiovasculares. Denotando en relación a este grupo, a un número mayor de mujeres que hombres, siendo la explicación de esta predominancia los roles de género que seguro deja en el grupo de mujeres la creencia o cultura de que el deporte es una actividad más propia de varones ya que son en apariencia los únicos con la capacidad de expresar resultados de fuerza, resistencia y poderío, mientras que en el caso femenino estas características se piensan no están vinculadas; no obstante esto es una idea errónea en la actualidad porque depende de la constancia de la practica 
independientemente del sexo para demostrar fortaleza física, siendo al final lo único relevante el practicar el deporte sin perjuicio alguno.

En Perú, existe desinterés de las instituciones referentes al deporte y como consecuencia, según Menezes (2016), los resultados demuestran poca competitividad de los deportistas peruanos al disputar un evento internacional. Esto se debe a que el deporte es visto como un tema de gasto y no como de inversión. De acuerdo a De la Torre (2018) se evidencia un alto nivel de deficiencia de gestión integral en la masificación del deporte. Asimismo, se observa deficiencias a nivel educación en donde se encuentra una pobre y escasa infraestructura de desarrollo del deporte y oportunidades que faciliten esta actividad para la formación de los niños a nivel regional y nacional. Resumiendo, falta la aplicación de una gestión integral por parte de las instituciones competentes, como también mejorar la inversión en el fortalecimiento de infraestructuras, medicina y equipamiento para los deportistas.

En el departamento de San Martín, se evidencia debilidad en el desarrollo y promoción de la actividad deportiva. En donde entidades como el Gobierno de la Región San Martín (GORESAM) y la Dirección Regional de Educación (DRE) tienen poco interés en la realización de actividades deportivas para lograr la masificación del deporte. Asimismo, puede observarse también, carencias en la gestión integral por parte de las instituciones antes mencionadas, evidenciado a sincronización entre ellas, para el desarrollo deportivo en San Martín, donde, presentan diversos problemas como la falta de infraestructura deportiva, corte de presupuesto para impulsar evento, torneos o campeonatos deportivos en nivel de educación primaria, segundaria y universitario, también, padecen de problemas en la proyección de desarrollo de jugadores de alto rendimiento, limitaciones al no disponer de estadística deportiva, como un sistema deportivo eficiente que este registrado en la base de datos en las instituciones, por lo que, resulta un alarmante problema en el desarrollo del deporte de San Martín, en la carente integración entre el Sistema Deportivo Nacional, con el Instituto Peruano del Deporte (IPD) San Martín como órgano rector y articulado integralmente con el Gobierno de la Región San Martín (GRSM) y la Dirección Regional de Educación (DRE). 


\section{Formulación del problema}

- ¿De qué manera la gestión integral contribuye al desarrollo deportivo en la región San Martín, 2020?

Como objetivo se propuso: Diseñar una propuesta de gestión integral que contribuya al desarrollo deportivo en la región de San Martín, 2020.

\section{MATERIAL Y MÉTODOS}

\section{Diseño de estudio}

Corresponde al tipo básico, la cual se caracteriza por la inexistencia de manipulación de variables deliberadamente, enfocándose en la descripción de los hechos y fenómenos en su estado natural.

Pertenece al diseño descriptivo propositivo. Asimismo, este diseño se caracteriza por tener el siguiente esquema:

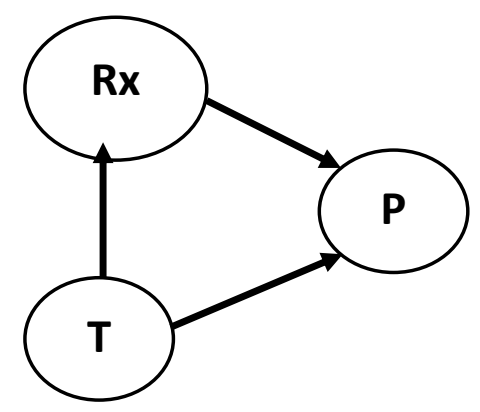

\section{Dónde:}

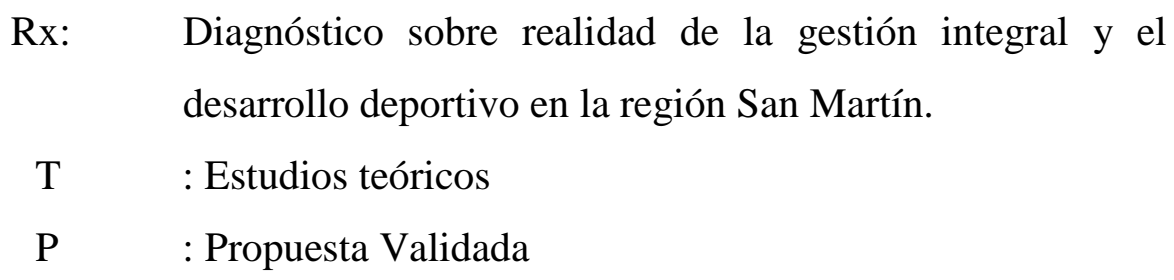

\section{Procedimiento de recolección de datos}

Según la finalidad del estudio de diseñar una propuesta de gestión integral para el desarrollo deportivo en la región San Martín, se solicitará permiso a las autoridades del gobierno regional. Mediante la autorización y el consentimiento de su participación se procederá a aplicar una encuesta con la finalidad de recolectar la información brindada por los participantes.

\section{Técnicas de análisis de datos}

Después de la recolección de los datos brindada por los participantes, estas serán digitalizadas para ser procesadas mediante software estadístico. Mediante la estadística 
descriptiva analizaremos la situación actual del desarrollo deportivo y en base a ello se diseñará una propuesta de gestión integral para su mejora, la cual será validada por expertos en gestión integral y desarrollo deportivo.

\section{RESULTADOS}

Los resultados del presente estudio se presentan a través de tablas, en los cuales se hallan detallados los artículos tomados, los mismos que corresponden a revistas indexadas, y que han servido como insumo del estudio, estos son: 


\section{Tabla 1. Estudios sobre gestión integral}

\section{DATOS}

\begin{tabular}{|c|c|c|c|c|c|}
\hline 1. Autor & Año & \multicolumn{2}{|c|}{ Nombre } & Revista & Vol. y núm. \\
\hline $\begin{array}{l}\text { Kathy Babiak, } \\
\text { Lucie Thibault } \\
\text { and Annick } \\
\text { Willem }\end{array}$ & 2017 & $\begin{array}{l}\text { Investigación ca } \\
\text { relaciones intero } \\
\text { gestión del depor } \\
\text { perspectivas de }\end{array}$ & $\begin{array}{l}\text { ráfica sobre las } \\
\text { izacionales en la } \\
\text { anorama actual y } \\
\text { stigación futura }\end{array}$ & $\frac{\text { https://doi.org/10.1123/jsm.2017-0099 }}{\text { Journal of Sport Management }}$ & $32(3)$ \\
\hline \multicolumn{6}{|c|}{ CONTENIDO } \\
\hline Tipo y diseño & $\begin{array}{l}\text { Población y } \\
\text { Muestra }\end{array}$ & Instrumentos & Aspectos éticos & Resultados & Conclusión \\
\hline $\begin{array}{c}\text { Básica, no } \\
\text { experimental }\end{array}$ & $\begin{array}{c}162 \\
\text { artículos }\end{array}$ & $\begin{array}{c}\text { Guía de análisis } \\
\text { documentario }\end{array}$ & $\begin{array}{l}\text { Presentación de } \\
\text { información } \\
\text { objetiva }\end{array}$ & $\begin{array}{l}\text { Los resultados revelaron un mayor énfasis en } \\
\text { las dimensiones de composición, estructura y } \\
\text { proceso de los IOR (Relaciones } \\
\text { interorganizacionales). }\end{array}$ & $\begin{array}{l}\text { Las diversas perspectivas, preguntas } \\
\text { y contextos explorados } \\
\text { contribuyeron a la riqueza del } \\
\text { conocimiento en gestión, además del } \\
\text { consenso sobre las teorías tratadas, } \\
\text { conceptos y marcos útiles para } \\
\text { evaluar los IOR entre las } \\
\text { organizaciones deportivas }\end{array}$ \\
\hline
\end{tabular}

Ciencia Latina Revista Científica Multidisciplinar, Ciudad de México, México.

ISN 2707-2207 / ISSN 2707-2215 (en línea), julio-agosto, 2021, Volumen 5, Número 4. https://doi.org/10.37811/cl_rcm.v5i4.789 p.6629 


\section{DATOS}

2. Autor

Año

Nombre

Gerencia Deportiva para la

Masificación Deportiva Planificada y

Reyes, L.

(2016)
Ejecutada por las Organizaciones

Sociales. Críticas a Modelos,

Experiencias y Propuesta de

Acompañamiento Metodológico

\section{CONTENIDO}

\begin{tabular}{|c|c|c|c|c|c|}
\hline Tipo y diseño & $\begin{array}{c}\text { Población y } \\
\text { muestra }\end{array}$ & Instrumentos & Aspectos éticos & Resultados & Conclusión \\
\hline $\begin{array}{l}\text { Básica, no } \\
\text { experimental }\end{array}$ & $\begin{array}{c}300 \text { consultados } \\
\text { entre } \\
\text { promotores, } \\
\text { organizadores } \\
\text { deportivos. }\end{array}$ & $\begin{array}{c}\text { Guía de análisis } \\
\text { documentario }\end{array}$ & $\begin{array}{c}\text { Presentación de } \\
\text { información } \\
\text { objetiva }\end{array}$ & $\begin{array}{l}\text { La ayuda con la plausibilidad razonable y elegida de } \\
\text { ofrecerla de manera eficiente a los anunciantes y pioneros } \\
\text { sociales asociados a la acción física y de desgaste y con } \\
\text { la alternativa de conectar tal sugerencia por medio de una } \\
\text { propuesta de expansión de la universidad enfocado en las } \\
\text { redes y sus animadores hacedores de un juegos modelo } \\
\text { social y en los límites de las asociaciones y } \\
\text { organizaciones emergentes. }\end{array}$ & $\begin{array}{l}\text { La masificación deportiva } \\
\text { planificada por } \\
\text { organizaciones sociales } \\
\text { de las comunidades son } \\
\text { experiencias de } \\
\text { Auto sustentabilidad, } \\
\text { participación, } \\
\text { intercambio, trabajo } \\
\text { facultativo } \\
\text { perseverancia } \\
\text { conservar en el espacio y } \\
\text { tiempo las actividades } \\
\text { deportivas. }\end{array}$ \\
\hline
\end{tabular}

Ciencia Latina Revista Científica Multidisciplinar, Ciudad de México, México. 


\section{DATOS}

\begin{tabular}{cc|cc}
\hline 3. Autor & Año & \multicolumn{1}{c}{ Nombre } & Revista donde su ubica la publicación \\
JonWelty & & Desafíos y $\quad$ estrategias para & $\underline{\text { https://doi.org/10.1016/j.smr.2017.06.002 }}$ \\
$\begin{array}{c}\text { Peachey, } \\
\text { AdamCohen, }\end{array}$ & 2018 & $\begin{array}{l}\text { construir y mantener asociaciones } \\
\text { interinstitucionales en el deporte } \\
\text { NariShina y }\end{array}$ & para el desarrollo y la paz \\
Bruno Fusaroa & & Sport Management Review & \\
\hline
\end{tabular}

\section{CONTENIDO}

\begin{tabular}{|c|c|c|c|c|c|}
\hline Tipo y diseño & $\begin{array}{c}\text { Población y } \\
\text { muestra }\end{array}$ & Instrumentos & Aspectos éticos & Resultados & Conclusión \\
\hline $\begin{array}{ll}\text { Básica, } & \text { no } \\
\text { experimental } & \end{array}$ & $\begin{array}{l}29 \\
\text { organizaciones }\end{array}$ & $\begin{array}{l}\text { Guía de análisis } \\
\text { documentario }\end{array}$ & $\begin{array}{l}\text { Presentación de } \\
\text { información } \\
\text { objetiva }\end{array}$ & $\begin{array}{l}\text { Los desafíos comunes encontrados en } 29 \text { organizaciones } \\
\text { incluyeron la competencia por los recursos, el } \\
\text { escepticismo del deporte como una herramienta de } \\
\text { desarrollo, relaciones de poder desiguales, objetivos } \\
\text { desalineados y desvíos de la misión, y problemas de } \\
\text { implementación. }\end{array}$ & $\begin{array}{l}\text { Las estrategias incluyeron } \\
\text { centrarse en la } \\
\text { construcción de relaciones } \\
\text { y redes, demostrar los } \\
\text { beneficios para el socio, } \\
\text { comenzar de a poco y } \\
\text { luego diversificar, } \\
\text { mantenerse enfocado en la } \\
\text { misión y los objetivos, } \\
\text { involucrar al socio y tratar } \\
\text { la asociación como una } \\
\text { relación comercial. }\end{array}$ \\
\hline
\end{tabular}

Ciencia Latina Revista Científica Multidisciplinar, Ciudad de México, México. 


\section{DATOS}

4. Autor Año $\quad$ Nombre Revista

\begin{tabular}{|c|c|c|}
\hline $\begin{array}{c}\text { Ana Anaya- } \\
\text { Velasco }\end{array}$ & 2017 & $\begin{array}{l}\text { Modelo de salud y seguridad en el } \\
\text { trabajo con Gestión Integral para la } \\
\text { sustentabilidad de las organizaciones }\end{array}$ \\
\hline
\end{tabular}

(SSeTGIS)

\section{http://dx.doi.org/10.4067/S0718-}

$\underline{24492017000200095}$

Ción Publicada en la Revista

Ciencia \& Trabajo

\begin{tabular}{|c|c|c|c|c|c|}
\hline \multicolumn{6}{|c|}{ CONTENIDO } \\
\hline Tipo y diseño & Población y Muestra & Instrumentos & Aspectos éticos & Resultados & Conclusión \\
\hline $\begin{array}{l}\text { Básica, No } \\
\text { experimental }\end{array}$ & $\begin{array}{l}\text { Modelo aplicado a } 10 \\
\text { organizaciones de toda } \\
\text { magnitud en tres sectores } \\
\text { de la economía }\end{array}$ & $\begin{array}{l}\text { Guía de análisis } \\
\text { documentario }\end{array}$ & $\begin{array}{l}\text { Presentación de } \\
\text { información } \\
\text { objetiva }\end{array}$ & $\begin{array}{l}\text { Se elaboró el instrumento de tipo } \\
\text { cuantitativo en una página de contabilidad } \\
\text { para el completo reconocimiento } \\
\text { dependiendo de las pautas que funcionan } \\
\text { con la estimación de la consistencia como } \\
\text { un hallazgo y luego la mejora a medida } \\
\text { que se distribuyen los activos, al igual que } \\
\text { los diferentes arreglos para el surtido de } \\
\text { información. }\end{array}$ & $\begin{array}{l}\text { Es significativo que las } \\
\text { asociaciones cuenten con } \\
\text { amplias opciones de modelos } \\
\text { que les permitan alcanzar sus } \\
\text { objetivos y satisfacer su } \\
\text { objetivo principal de la manera } \\
\text { más ideal, potenciando ventajas } \\
\text { y disminuyendo daños y gastos. }\end{array}$ \\
\hline
\end{tabular}

Ciencia Latina Revista Científica Multidisciplinar, Ciudad de México, México.

ISN 2707-2207 / ISSN 2707-2215 (en línea), julio-agosto, 2021, Volumen 5, Número 4. https://doi.org/10.37811/cl rem.v5i4.789 p.6632 


\section{DATOS}

5. Autor Año Nombre Revista $\quad$ Vol. yúm.

Kozlov, A

Budarnikov, A

Zhuravleva, Yu

(2018)

Desarrollo del mercado de

The journal of social sciences research

Gonsales, S

servicios deportivos en las

https://www.elibrary.ru/item.asp?id=365

$\underline{44846}$

Lebdeva, O

\section{CONTENIDO}

\begin{tabular}{|c|c|c|c|c|c|}
\hline Tipo y diseño & $\begin{array}{c}\text { Población y } \\
\text { Muestra }\end{array}$ & Instrumentos & $\begin{array}{l}\text { Aspectos } \\
\text { éticos }\end{array}$ & Resultados & Conclusión \\
\hline $\begin{array}{c}\text { Tipo básica } \\
\text { No experimental }\end{array}$ & $\begin{array}{c}\text { El gobierno } \\
\text { ruso }\end{array}$ & $\begin{array}{l}\text { Análisis } \\
\text { documental } \\
\text { entrevista }\end{array}$ & $\begin{array}{c}\text { Presentación } \\
\text { de } \\
\text { información } \\
\text { objetiva }\end{array}$ & $\begin{array}{l}\text { El desarrollo del mercado de servicios } \\
\text { deportivos es crucial para el desarrollo } \\
\text { de la nación y su potencial humano y } \\
\text { requiere el uso de un enfoque sistemático } \\
\text { en todas las áreas de enfoque clave }\end{array}$ & $\begin{array}{l}\text { La gestión eficaz del movimiento de fitness / } \\
\text { deportes a nivel de regiones y empresas se } \\
\text { trata de asignar de manera competente los } \\
\text { fondos desembolsados para promover el } \\
\text { desarrollo del sector de servicios deportivos } \\
\text { privados de la nación. }\end{array}$ \\
\hline
\end{tabular}

Ciencia Latina Revista Científica Multidisciplinar, Ciudad de México, México.

ISN 2707-2207 / ISSN 2707-2215 (en línea), julio-agosto, 2021, Volumen 5, Número 4.

https://doi.org/10.37811/cl_rcm.v5i4.789 p.6633 


\section{DATOS}

\begin{tabular}{|c|c|c|c|c|}
\hline 6. Autor & Año & Nombre & Revista & Vol. y núm. \\
\hline $\begin{array}{l}\text { Alcántara, I., Garza, M., } \\
\text { López, A., Magaña, V., } \\
\text { Oropeza, O., Puente, S., } \\
\text { Rodríguez, D., } \\
\text { Lucatello, S., Ruiz, N., } \\
\text { Tena, R., Urzúa, M. y } \\
\text { Vázquez, G. }\end{array}$ & 2019 & $\begin{array}{l}\text { Gestión Integral de riesgos } \\
\text { de desastres en México: } \\
\text { reflexiones, retos y } \\
\text { propuestas } \\
\text { transformación de la Política } \\
\text { Pública desde la academia }\end{array}$ & $\begin{array}{l}\text { https://doi.org/10.14350/rig.59784 } \\
\text { Investigación publicada en la Revista } \\
\text { Investigaciones Geográficas }\end{array}$ & Num. 98 \\
\hline
\end{tabular}

\section{CONTENIDO}

\begin{tabular}{|c|c|c|c|c|c|}
\hline Tipo y diseño & $\begin{array}{c}\text { Población y } \\
\text { Muestra }\end{array}$ & Instrumentos & $\begin{array}{l}\text { Aspectos } \\
\text { éticos }\end{array}$ & Resultados & Conclusión \\
\hline Básica, no experimental & $\begin{array}{c}\text { Las } 32 \text { entidades } \\
\text { federativas de } \\
\text { México }\end{array}$ & $\begin{array}{c}\text { Guía de } \\
\text { análisis } \\
\text { documentario }\end{array}$ & $\begin{array}{c}\text { Presentación } \\
\text { de } \\
\text { información } \\
\text { objetiva }\end{array}$ & $\begin{array}{l}\text { Los productos de las tertulias de } \\
\text { debate, de la misma forma que las } \\
\text { sugerencias de fondo, terminaron en la } \\
\text { asociación del fórum denominado: } \\
\text { "Del Sistema Nacional de Protección } \\
\text { Civil al Sistema Nacional de Gestión } \\
\text { Integral del Riesgo de Desastres: } \\
\text { Desafíos, reflexiones y Propuestas de } \\
\text { cambio en la Academia", celebrada } \\
\text { dentro de la estructura de la } \\
\text { celebración de la fecha mundial de la } \\
\text { reducción del riesgo de desastres en el } \\
\text { año } 2018 \text {. }\end{array}$ & $\begin{array}{l}\text { Enmarcado en la metodología de Gestión } \\
\text { Integral del Riesgo de Desastres, un origen } \\
\text { similar de la normativa genérica de amparo } \\
\text { Civil, presenta dificultades críticas de } \\
\text { coordinación y cambios institucionales, } \\
\text { gerenciales, administrativos y monetarios, así } \\
\text { como de reconceptualización, para su } \\
\text { ejecución productiva como Política. Público. }\end{array}$ \\
\hline
\end{tabular}

Ciencia Latina Revista Científica Multidisciplinar, Ciudad de México, México.

ISN 2707-2207 / ISSN 2707-2215 (en línea), julio-agosto, 2021, Volumen 5, Número 4. https://doi.org/10.37811/cl rem.v5i4.789 p.6634 


\section{DATOS}

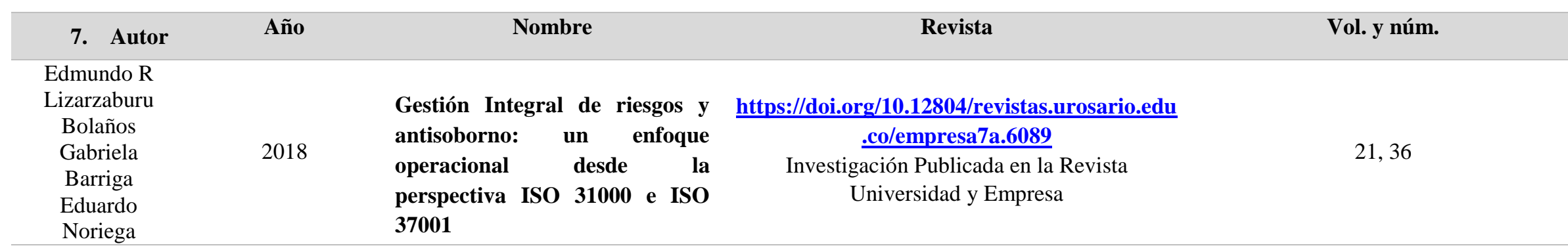

\section{CONTENIDO}

\begin{tabular}{ccccc} 
Tipo y diseño & $\begin{array}{c}\text { Población y } \\
\text { Muestra }\end{array}$ & Instrumentos & $\begin{array}{c}\text { Aspectos } \\
\text { éticos }\end{array}$ & Resultados \\
\hline Básica, no & 05 organizaciones & Guía de análisis & Presentación & Tener indicadores, por ejemplo, KPI ayuda a Las ventajas y ramificaciones de la \\
experimental & documentario & de & tener la opción de predecir fuentes de peligro, normativa iso 31000 , iso 37001 e iso \\
& & información & por lo que tener la opción de ajustar los avances integral 9001 han sido planteadas, \\
& & objetiva & de datos a los requisitos y propósitos de la examinadas y relacionadas con la \\
& & & organización es una labor compleja de dicho administración al azar desde una \\
& & & contexto. &
\end{tabular}

Ciencia Latina Revista Científica Multidisciplinar, Ciudad de México, México. 


\section{DATOS}

\begin{tabular}{|c|c|c|c|c|}
\hline 8. Autor & Año & Nombre & Revista & Vol. y núm. \\
\hline García I & & & 262/visionariodigital.v3i2.47 & \\
\hline $\begin{array}{l}\text { Narváez, C. y } \\
\text { Erazo, J. }\end{array}$ & 2019 & $\begin{array}{l}\text { Gestión Integral de activos fijos } \\
\text { con enfoque en el control } \\
\text { contable administrativo }\end{array}$ & $\begin{array}{l}\quad \underline{9} \\
\text { cada en la Revista Visionario } \\
\text { Digital }\end{array}$ & 3,2 \\
\hline
\end{tabular}

\section{CONTENIDO}

\begin{tabular}{|c|c|c|c|c|c|}
\hline Tipo y diseño & Población y Muestra & Instrumentos & $\begin{array}{l}\text { Aspectos } \\
\text { éticos }\end{array}$ & Resultados & Conclusión \\
\hline $\begin{array}{c}\text { Básica, no } \\
\text { experimental }\end{array}$ & $\begin{array}{l}5 \text { trabajadores } \\
\text { administrativos de la } \\
\text { Agrupación } \\
\text { Agroartesanal de } \\
\text { Productores de Palanda } \\
\text { y Chinchipe de Zamora } \\
\text { Chinchipe, Ecuador. }\end{array}$ & Cuestionario & $\begin{array}{c}\text { Presentación } \\
\text { de } \\
\text { información } \\
\text { objetiva }\end{array}$ & $\begin{array}{l}\text { Analizar el correcto uso, ejecución y mal uso } \\
\text { de los recursos fijos de la asociación a través } \\
\text { de normas o punteros dará signos de } \\
\text { efectividad funcional para caracterizar } \\
\text { metodologías y arreglos que desarrollen aún } \\
\text { más las medidas y de esta manera la ejecución } \\
\text { jerárquica. }\end{array}$ & $\begin{array}{l}\text { El recurso a los ejecutivos es un } \\
\text { instrumento fundamental para } \\
\text { aprovechar su límite introducido en } \\
\text { lo que respecta a la mejora } \\
\text { empresarial, de igual manera } \\
\text { percibir su kilometraje, al igual que } \\
\text { al cerrar su periodo de vida para } \\
\text { sanear los activos útiles que } \\
\text { realmente tiene la organización.. }\end{array}$ \\
\hline
\end{tabular}

Ciencia Latina Revista Científica Multidisciplinar, Ciudad de México, México. ISN 2707-2207 / ISSN 2707-2215 (en línea), julio-agosto, 2021, Volumen 5, Número 4. https://doi.org/10.37811/cl rem.v5i4.789 p.6636 


\section{DATOS}

9. Autor Año Nombre Revista núm.

Summerley, Rory

(2020)
El Desarrollo del Deporte: Un Análisis Comparativo de la Institucionalización Temprana de los Deportes Tradicionales y las E-Sports

\author{
Games and Culture \\ https://journals.sagepub.com/doi/full/10.1177/155 \\ $\underline{5412019838094}$
}

$15(1)$

\section{CONTENIDO}

\begin{tabular}{|c|c|c|c|c|c|}
\hline Tipo y diseño & $\begin{array}{c}\text { Población y } \\
\text { Muestra }\end{array}$ & Instrumentos & $\begin{array}{l}\text { Aspectos } \\
\text { éticos }\end{array}$ & Resultados & Conclusión \\
\hline $\begin{array}{l}\text { Tipo básica no } \\
\text { experimental } \\
\text { comparativo }\end{array}$ & 19 instituciones & $\begin{array}{c}\text { Análisis } \\
\text { documental }\end{array}$ & $\begin{array}{l}\text { Presentación } \\
\text { de } \\
\text { información } \\
\text { objetiva }\end{array}$ & $\begin{array}{l}\text { Se identificaron similitudes y diferencias entre } \\
\text { la institucionalización de los deportes } \\
\text { tradicionales entre la mitad y el final del siglo } \\
\text { XIX y los deportes electrónicos desde } \\
\text { mediados de la década de } 1990 \text { en adelante, y } \\
\text { gran parte de esto tiene que ver con sus } \\
\text { contextos históricos }\end{array}$ & $\begin{array}{l}\text { Las instituciones deportivas de } \\
\text { ambos tipos establecen una } \\
\text { filosofía moral que orienta el } \\
\text { carácter del deporte y su } \\
\text { comunidad como parte del proceso } \\
\text { de institucionalización. Mediante la } \\
\text { propagación de su juego a través de } \\
\text { eventos promocionales. }\end{array}$ \\
\hline
\end{tabular}

Ciencia Latina Revista Científica Multidisciplinar, Ciudad de México, México. ISN 2707-2207 / ISSN 2707-2215 (en línea), julio-agosto, 2021, Volumen 5, Número 4. https://doi.org/10.37811/cl_rcm.v5i4.789 p.6637 


\section{Tabla 2. Estudios sobre desarrollo deportivo}

DATOS

\begin{tabular}{|c|c|c|c|c|c|}
\hline 1. Autor & Año & \multicolumn{2}{|c|}{ Nombre } & Revista & Vol. y núm. \\
\hline $\begin{array}{l}\text { Evan Gwartz y } \\
\text { Kirsty Spence }\end{array}$ & 2020 & \multicolumn{2}{|c|}{$\begin{array}{l}\text { Capitalismo consciente y deporte: } \\
\text { Explorando un propósito superior } \\
\text { en una organización deportiva } \\
\text { profesional }\end{array}$} & $\frac{\text { https://doi.org/10.1016/j.smr.2019.09.002 }}{\text { Sport Management Review }}$ & 23,4 \\
\hline \multicolumn{6}{|c|}{ CONTENIDO } \\
\hline Tipo y diseño & $\begin{array}{c}\text { Población y } \\
\text { Muestra }\end{array}$ & Instrumentos & Aspectos éticos & Resultados & Conclusión \\
\hline $\begin{array}{l}\text { Básica, no } \\
\text { experimental }\end{array}$ & 13 líderes & $\begin{array}{l}\text { Guía de análisis } \\
\text { documentario, } \\
\text { entrevistas }\end{array}$ & $\begin{array}{l}\text { Presentación de } \\
\text { información } \\
\text { objetiva }\end{array}$ & $\begin{array}{l}\text { los investigadores de gestión proporcionaron evidencia de que la } \\
\text { comunicación de los líderes organizacionales sobre el propósito } \\
\text { superior de una organización beneficia profundamente el } \\
\text { desempeño organizacional. }\end{array}$ & $\begin{array}{l}\text { los líderes como los } \\
\text { académicos tienen la } \\
\text { oportunidad } \\
\text { reflexionar } \\
\text { colectivamente y definir } \\
\text { el propósito de una } \\
\text { organización deportiva } \\
\text { profesional }\end{array}$ \\
\hline
\end{tabular}

Ciencia Latina Revista Científica Multidisciplinar, Ciudad de México, México. 


\section{DATOS}

\begin{tabular}{|c|c|c|c|c|c|}
\hline 2. Autor & Año & \multicolumn{2}{|c|}{ Nombre } & Revista & Vol. y núm. \\
\hline Javier Reyes & 2020 & $\begin{array}{l}\text { Sport omniv } \\
\text { stratification of s } \\
\text { Chile }\end{array}$ & $\begin{array}{l}\text { rism: } \begin{array}{r}\text { Social } \\
\text { orts practice in }\end{array}\end{array}$ & $\frac{\text { https://doi.org/10.1177\%2F1012690220959102 }}{\text { International Review for the Sociology of Sport }}$ & 1,1 \\
\hline \multicolumn{6}{|c|}{ CONTENIDO } \\
\hline Tipo y diseño & $\begin{array}{l}\text { Población y } \\
\text { Muestra }\end{array}$ & Instrumentos & $\begin{array}{l}\text { Aspectos } \\
\text { éticos }\end{array}$ & Resultados & Conclusión \\
\hline $\begin{array}{l}\text { Básica, no } \\
\text { experimental }\end{array}$ & $\begin{array}{l}5511 \\
\text { jóvenes } \\
\text { deportivos }\end{array}$ & $\begin{array}{l}\text { Guía de análisis } \\
\text { documentario, } \\
\text { análisis de clases }\end{array}$ & $\begin{array}{l}\text { Presentación de } \\
\text { información } \\
\text { objetiva }\end{array}$ & $\begin{array}{l}\text { Los resultados apoyan la literatura actual sobre la } \\
\text { participación deportiva, encontrando evidencia a favor } \\
\text { tanto de la hipótesis de la homología como de la } \\
\text { hipótesis omnívora-unívora. }\end{array}$ & $\begin{array}{l}\text { Mediante las regresiones } \\
\text { logísticas y multinomiales al } \\
\text { relacionar los tipos de prácticas } \\
\text { deportivas con sus determinantes } \\
\text { sociales, concluye que los } \\
\text { patrones de estratificación social } \\
\text { en la práctica deportiva en Chile } \\
\text { se reconfiguran en el ámbito del } \\
\text { deporte. }\end{array}$ \\
\hline
\end{tabular}

Ciencia Latina Revista Científica Multidisciplinar, Ciudad de México, México. 


\section{DATOS}

\section{Autor}

Año

Nombre

Análisis del Desarrollo Deportivo

en la Regencia Bima desde la

Perspectiva Epistemológica https://dx.doi.org/10.2991/assehr.k.200620.057 Advances in

Social Science, Education and Humanities Research
Vol. y núm.

\section{CONTENIDO}

\begin{tabular}{|c|c|c|c|c|c|}
\hline Tipo y diseño & $\begin{array}{l}\text { Población y } \\
\text { Muestra }\end{array}$ & Instrumentos & Aspectos éticos & Resultados & Conclusión \\
\hline $\begin{array}{l}\text { Básica, no } \\
\text { experimental }\end{array}$ & 13 líderes & $\begin{array}{l}\text { Guía de análisis } \\
\text { documentario, } \\
\text { análisis normativo }\end{array}$ & $\begin{array}{l}\text { Presentación de } \\
\text { información } \\
\text { objetiva }\end{array}$ & $\begin{array}{l}\text { El deporte es un importante factor y parte de los esfuerzos para } \\
\text { mejorar la calidad de vida del humano intentando fortalecer el } \\
\text { conocimiento en mejorar el desarrollo deportivo } \\
\text { El desarrollo deportivo es una abstracción para crear personas } \\
\text { competitivas, íntegras y sobresalientes. }\end{array}$ & $\begin{array}{l}\text { Los deportes se pueden } \\
\text { hacer como ejercicios, } \\
\text { educación, } \\
\text { entretenimiento, } \\
\text { recreación, } \\
\text { profesión, } \\
\text { política, } \\
\text { industria y varios otros, } \\
\text { aspectos de } \\
\text { cultura humana. }\end{array}$ \\
\hline
\end{tabular}

Ciencia Latina Revista Científica Multidisciplinar, Ciudad de México, México. 


\section{DATOS}

\begin{tabular}{|c|c|c|c|c|}
\hline 4. Autor & Año & Nombre & Revista & Vol. y núm. \\
\hline $\begin{array}{l}\text { Di Palma, D., Tafuri, } \\
\text { D. Ascione, A. y } \\
\text { Raiola, G. }\end{array}$ & 2018 & $\begin{array}{l}\text { Desarrollo social, turístico y } \\
\text { educativo a través del deporte }\end{array}$ & $\begin{array}{l}\text { https://doi.org/10.7752/jpes.2018.s167 } \\
\text { Journal of Physical Education and Sport }\end{array}$ & 18,1 \\
\hline
\end{tabular}

\section{CONTENIDO}

\begin{tabular}{|c|c|c|c|c|c|c|}
\hline Tipo y diseño & & $\begin{array}{c}\text { Población y } \\
\text { Muestra }\end{array}$ & Instrumentos & Aspectos éticos & Resultados & Conclusión \\
\hline $\begin{array}{l}\text { Básica, } \\
\text { experimental }\end{array}$ & no & 34 revistas & $\begin{array}{l}\text { Guía de análisis } \\
\text { documentario }\end{array}$ & $\begin{array}{l}\text { Presentación de } \\
\text { información } \\
\text { objetiva }\end{array}$ & $\begin{array}{l}\text { Se demostró cómo a través de un proceso evolutivo en el } \\
\text { tiempo, el deporte ha llegado a afectar positivamente a } \\
\text { otros contextos, incluyendo ciertamente la educación y la } \\
\text { escuela, la industria del turismo y lo concerniente } \\
\text { cuestiones socioeconómicas. }\end{array}$ & $\begin{array}{l}\text { Enfatizar la necesidad de } \\
\text { administrar el sistema } \\
\text { deportivo desde una } \\
\text { perspectiva gerencial. } \\
\text { a través de procesos } \\
\text { operativos y de toma de } \\
\text { decisiones basados en } \\
\text { criterios económicos de } \\
\text { eficiencia y eficacia }\end{array}$ \\
\hline & & & & & & $\begin{array}{l}\text { que puede asegurar } \\
\text { resultados satisfactorios en } \\
\text { términos de costo. }\end{array}$ \\
\hline
\end{tabular}

Ciencia Latina Revista Científica Multidisciplinar, Ciudad de México, México. ISN 2707-2207 / ISSN 2707-2215 (en línea), julio-agosto, 2021, Volumen 5, Número 4. https://doi.org/10.37811/cl_rcm.v5i4.789 p.6641 


\section{DATOS}

$\begin{array}{llll}\text { 5. Autor Nombre } & \text { Revista } & \text { Vol. y núm. }\end{array}$

$\begin{array}{cclcc}\text { Mujika, I., Halson, } & & \text { Un enfoque multifactorial e } & \text { https://doi.org/10.1123/ijspp.2018-0093 } \\ \text { S., Burke, L., } & 2018 & \text { integrado de la periodización para } & \text { International Journal of Sports Physiology and } \\ \text { Balagué, G. y } & & \text { un rendimiento óptimo en } & \underline{\text { Performance }} \\ \text { Farrow, D. } & & \text { deportes individuales y de equipo } & \end{array}$

\section{CONTENIDO}

\begin{tabular}{|c|c|c|c|c|c|}
\hline Tipo y diseño & $\begin{array}{c}\text { Población y } \\
\text { Muestra }\end{array}$ & Instrumentos & Aspectos éticos & Resultados & Conclusión \\
\hline $\begin{array}{l}\text { Básica, } \\
\text { experimental }\end{array}$ & 188 revistas & $\begin{array}{l}\text { Guía de análisis } \\
\text { documentario }\end{array}$ & $\begin{array}{l}\text { Presentación de } \\
\text { información } \\
\text { objetiva }\end{array}$ & $\begin{array}{l}\text { La periodización física como la estratégica son } \\
\text { herramientas útiles para manejar el horario de viaje } \\
\text { pesado, la fatiga y las lesiones que ocurren durante } \\
\text { una temporada competitiva. Las intervenciones de } \\
\text { recuperación deben ser periódicas (es decir, retenidas } \\
\text { o enfatizadas) para influir en la adaptación y el } \\
\text { rendimiento del entrenamiento agudo y crónico. }\end{array}$ & $\begin{array}{l}\text { La sincronización en relación con } \\
\text { el ejercicio y como parte de la } \\
\text { periodización del calendario de } \\
\text { entrenamiento y competición de } \\
\text { un atleta también pueden } \\
\text { promover adaptaciones } \\
\text { fisiológicas y capacidad de } \\
\text { rendimiento. Las habilidades } \\
\text { psicológicas son un componente } \\
\text { central del rendimiento deportivo } \\
\text { y su periodización debe satisfacer } \\
\text { las necesidades individuales de } \\
\text { cada atleta y las necesidades del } \\
\text { equipo. }\end{array}$ \\
\hline
\end{tabular}

Ciencia Latina Revista Científica Multidisciplinar, Ciudad de México, México.

ISN 2707-2207 / ISSN 2707-2215 (en línea), julio-agosto, 2021, Volumen 5, Número 4. https://doi.org/10.37811/cl rem.v5i4.789 p.6642 


\section{DATOS}

\section{Autor}

Mathew Dowling

, Becca Leopkey and Lee Smith
Año

2018

Gobernanza en el deporte: una
revisión de alcance
Revista

https://doi.org/10.1123/jsm.2018-0032

Journal of Sport Management
Vol. y núm.

\section{2,5}

\section{CONTENIDO}

\begin{tabular}{|c|c|c|c|c|c|}
\hline Tipo y diseño & $\begin{array}{c}\text { Población y } \\
\text { Muestra }\end{array}$ & Instrumentos & Aspectos éticos & Resultados & Conclusión \\
\hline $\begin{array}{l}\text { Básica, no } \\
\text { experimental, } \\
\text { revisión } \\
\text { bibliográfica }\end{array}$ & $\begin{array}{l}243 \\
\text { artículos }\end{array}$ & $\begin{array}{l}\text { Guía de análisis } \\
\text { documentario }\end{array}$ & $\begin{array}{l}\text { Presentación de } \\
\text { información } \\
\text { objetiva }\end{array}$ & $\begin{array}{l}\text { El análisis de frecuencia revela un aumento notable en la } \\
\text { investigación sobre gobernanza deportiva en los últimos } \\
\text { años con una gran cantidad de estudios no empíricos } \\
\text { centrados en el área sin fines lucrativos. El análisis temático } \\
\text { se basa y amplía las tres nociones de gobernanza de Henry } \\
\text { y Lee e identifica temas relacionados con la gobernanza } \\
\text { deportiva, contextos de investigación y cuestiones sociales }\end{array}$ & \begin{tabular}{lcr} 
Las tres & formas de \\
gobernanza & \multicolumn{2}{c}{ (organizativa, } \\
sistémica y & política) han \\
contribuido a & nuestra \\
comprensión & de & la \\
gobernanza deportiva, pero se \\
necesitan más investigaciones \\
empíricas y teóricas.
\end{tabular} \\
\hline
\end{tabular}

Ciencia Latina Revista Científica Multidisciplinar, Ciudad de México, México. 


\section{DATOS}

\section{Autor}

Año

Nombre

Milena M.

Parent y Russell Hoye
El impacto de los principios de gobernanza en las prácticas y el rendimiento de las organizaciones

deportivas: una revisión sistemática

\section{CONTENIDO}

https://doi.org/10.1080/23311886.2018.1503578

Cogent Social Sciences
Vol. y núm.

\begin{tabular}{|c|c|c|c|c|c|}
\hline Tipo y diseño & $\begin{array}{l}\text { Población } \\
\text { y Muestra }\end{array}$ & Instrumentos & Aspectos éticos & Resultados & Conclusión \\
\hline $\begin{array}{l}\text { Básica, no } \\
\text { experimental } \\
\text { revisión } \\
\text { bibliográfica }\end{array}$ & $\begin{array}{l}\text { Población } \\
2155 \\
\text { Muestra: } 19\end{array}$ & $\begin{array}{l}\text { Guía de análisis } \\
\text { documentario }\end{array}$ & $\begin{array}{l}\text { Presentación de } \\
\text { información } \\
\text { objetiva }\end{array}$ & $\begin{array}{l}\text { Países principalmente desarrollados que examinaban el tema, a } \\
\text { menudo usando estudios de casos como un medio para explorar } \\
\text { el tema. Aunque el vínculo entre la estructura de la junta y el } \\
\text { desempeño organizacional se ha encontrado empíricamente, el } \\
\text { vínculo entre otros principios de gobernanza y el desempeño } \\
\text { organizacional sigue siendo deficiente. }\end{array}$ & $\begin{array}{l}\text { Existe una clara } \\
\text { necesidad de que tanto la } \\
\text { comunidad deportiva } \\
\text { internacional como los } \\
\text { investigadores } \\
\text { desarrollan un conjunto } \\
\text { acordado de principios } \\
\text { de gobernanza y un } \\
\text { lenguaje relevante a } \\
\text { nivel internacional, } \\
\text { nacional, provincial / } \\
\text { estatal y local }\end{array}$ \\
\hline
\end{tabular}

Ciencia Latina Revista Científica Multidisciplinar, Ciudad de México, México. ISN 2707-2207 / ISSN 2707-2215 (en línea), julio-agosto, 2021, Volumen 5, Número 4. https://doi.org/10.37811/cl rem.v5i4.789 p.6644 


\section{DATOS}

\begin{tabular}{|c|c|c|c|c|}
\hline 8. Autor & Año & Nombre & Revista & Volumen y número \\
\hline $\begin{array}{l}\text { Prato, 1., } \\
\text { Alcaraz, s., } \\
\text { Ramis, Y. y } \\
\text { Torregrossa, M. }\end{array}$ & 2020 & $\begin{array}{c}\text { Experiencias del entorno } \\
\text { deportivo de origen al } \\
\text { asesorar a esgrimistas } \\
\text { migrantes }\end{array}$ & $\begin{array}{l}: \underline{\text { https://doi.org/10.11144/Javerianacali.PPSI18- }} \\
\underline{2 . \text { eedo }} \\
\text { Investigación Publicada en la Revista Pensamiento } \\
\text { Psicológico }\end{array}$ & 18,02 \\
\hline
\end{tabular}

\begin{tabular}{|c|c|c|c|c|c|}
\hline Tipo y diseño & Población y Muestra & Instrumentos & $\begin{array}{l}\text { Aspectos } \\
\text { éticos }\end{array}$ & Resultados & Conclusión \\
\hline $\begin{array}{l}\text { Básica, no } \\
\text { experimental } \\
\text { revisión } \\
\text { bibliográfica }\end{array}$ & $\begin{array}{l}\text { Cuatro trabajadores } \\
\text { de la salud y cinco } \\
\text { entrenadores }\end{array}$ & Cuestionario & $\begin{array}{l}\text { Presentación } \\
\text { de información } \\
\text { objetiva }\end{array}$ & $\begin{array}{l}\text { Está comprobado que seguir el progreso social de } \\
\text { los esgrimistas transitorios impacta la prosperidad } \\
\text { y los elementos directivos de los mentores y } \\
\text { expertos en bienestar. Se crearon dos temas } \\
\text { focales: cuidado y caminar y descansar. }\end{array}$ & $\begin{array}{l}\text { La migración en el deporte } \\
\text { plantea nuevas dificultades al } \\
\text { grupo multidisciplinar que } \\
\text { acompaña y exhorta a los } \\
\text { competidores, a todo tipo de } \\
\text { personas. Se proponen } \\
\text { sugerencias con los pies en la } \\
\text { tierra para mentores y expertos } \\
\text { en bienestar, en vista de la } \\
\text { práctica social de la Psicología } \\
\text { Cultural que se aplica al } \\
\text { desgaste. }\end{array}$ \\
\hline
\end{tabular}

Ciencia Latina Revista Científica Multidisciplinar, Ciudad de México, México. 


\section{DATOS}

\begin{tabular}{|c|c|c|c|c|c|}
\hline 9. Autor & Año & \multicolumn{2}{|c|}{ Nombre } & Revista & Vol. y núm. \\
\hline $\begin{array}{c}\text { Arronte, J. y } \\
\text { Ferro, B. }\end{array}$ & 2020 & \multicolumn{2}{|c|}{$\begin{array}{l}\text { El proceso de iniciación } \\
\text { deportiva escolar en voleibol: } \\
\text { una mirada de renovación de } \\
\text { aprendizaje }\end{array}$} & $\begin{array}{l}\frac{\underline{\text { http://podium.upr.edu.cu/index.php/podium/article/ }}}{\underline{\text { view/912 }}} \\
\text { Investigación Publicada en la Revista de Ciencia y } \\
\text { Tecnología en la Cultura Física }\end{array}$ & \multirow[t]{2}{*}{15,1} \\
\hline \multicolumn{5}{|r|}{ CONTENIDO } & \\
\hline Tipo y diseño & $\begin{array}{c}\text { Población y } \\
\text { Muestra }\end{array}$ & Instrumentos & $\begin{array}{l}\text { Aspectos } \\
\text { éticos }\end{array}$ & Resultados & Conclusión \\
\hline $\begin{array}{l}\text { Básica, no } \\
\text { experimental } \\
\text { revisión } \\
\text { bibliográfica }\end{array}$ & $\begin{array}{l}12 \text { atletas y } 8 \\
\text { directivos y } \\
\text { metodólogos. }\end{array}$ & Cuestionario & $\begin{array}{l}\text { Presentación } \\
\text { de } \\
\text { información } \\
\text { objetiva }\end{array}$ & $\begin{array}{l}\text { La sistematización hizo que lo hiciera concebible } \\
\text { para decidir patrones en la mejora del ítem, } \\
\text { caracterizarlo y construir tres medidas para su } \\
\text { investigación (intelectual procedimental, el tablero } \\
\text { de trabajo metodológico y axiológico) con sus } \\
\text { definiciones individuales, indicar cualidades y } \\
\text { falencias que lo describen. en la organización de los } \\
\text { juegos de referencia, al igual que la combinación de } \\
\text { resultados analíticos en la base de sus } \\
\text { normalidades. }\end{array}$ & $\begin{array}{l}\text { Los análisis sobre el proceso de } \\
\text { iniciación deportiva se perfilan hacia la } \\
\text { conceptualización, aseguramiento de sus } \\
\text { características, estándares y premisas, la } \\
\text { incorporación de componentes de } \\
\text { preparación y la importante conexión } \\
\text { con el entorno familiar y el ámbito local } \\
\text { en el que se trabaja, potenciando la } \\
\text { mejora de diferentes límites. que } \\
\text { satisfagan las necesidades } \\
\text { inspiraciones individuales y sociales del } \\
\text { competidor. }\end{array}$ \\
\hline
\end{tabular}

Ciencia Latina Revista Científica Multidisciplinar, Ciudad de México, México. 


\section{DATOS}

\begin{tabular}{|c|c|c|c|c|}
\hline 10. Autor & Año & Nombre & Revista & Vol. y núm. \\
\hline $\begin{array}{l}\text { Maqueira G, } \\
\text { Brito E, Recalde } \\
\text { A, Arévalo J, } \\
\text { Sáenz J y } \\
\text { Sandoval, M. }\end{array}$ & 2017 & $\begin{array}{l}\text { Tendencias y manifestaciones } \\
\text { del temperamento relación con } \\
\text { el desempeño social y físico- } \\
\text { deportivo en estudiantes }\end{array}$ & $\begin{array}{l}\underline{\text { https://www.medigraphic.com/cgi- }} \\
\underline{\text { bin/new/resumen.cgi?IDARTICULO=79008 }} \\
\text { Investigación Publicada en la Revista Cubana de } \\
\text { Investigaciones Biomédicas }\end{array}$ & 36,02 \\
\hline \multicolumn{5}{|c|}{ CONTENIDO } \\
\hline Tipo y diseño & $\begin{array}{c}\text { Población y } \\
\text { Muestra }\end{array}$ & Aspectos éticos & Resultados & Conclusión \\
\hline $\begin{array}{l}\text { Básica, no } \\
\text { experimental } \\
\text { revisión } \\
\text { bibliográfica }\end{array}$ & $\begin{array}{l}\text { Son estudiados } 19 \\
\text { estudiantes de } \\
\text { ambos sexos que } \\
\text { participan en las } \\
\text { clases, a quienes se } \\
\text { les aplicó un test } \\
\text { para medir el } \\
\text { temperamento. }\end{array}$ & $\begin{array}{l}\text { Presentación de } \\
\text { información } \\
\text { objetiva }\end{array}$ & $\begin{array}{l}\text { Se consiguió la evaluación de las tendencias } \\
\text { centrales y expresiones del temperamento que } \\
\text { identificaban al estudiantado, fue elaborada la } \\
\text { estrategia metodológica docente que posibilitó } \\
\text { guiar al colectivo acerca de la optimización de las } \\
\text { tendencias del temperamento que tenían, y } \\
\text { controlar de forma adecuada las cualidades } \\
\text { negativas que se expresan en su temperamento. }\end{array}$ & $\begin{array}{l}\text { Evaluar el temperamento del } \\
\text { colectivo partícipe, consigue } \\
\text { influir a través de la estrategia } \\
\text { metodológica docente en } \\
\text { desempeño mejor en el ámbito } \\
\text { deportivo y físico, así como en } \\
\text { el social del estudiantado. }\end{array}$ \\
\hline
\end{tabular}

Ciencia Latina Revista Científica Multidisciplinar, Ciudad de México, México. 
Tabla 3. Estudios sobre gestión integral y desarrollo deportivo

\begin{tabular}{|c|c|c|c|c|}
\hline \multicolumn{5}{|c|}{ DATOS } \\
\hline 1. Autor & Año & Nombre & Revista & Vol. y núm. \\
\hline $\begin{array}{l}\text { Thanh, Phan } \\
\text { Viet, Tran }\end{array}$ & (2018) & $\begin{array}{l}\text { La gestión de los recursos } \\
\text { deportivos en la } \\
\text { Nacional Universidad } \\
\text { Vietnam }\end{array}$ & $\begin{array}{l}\begin{array}{l}\text { European Journal of Physical Educatión and Sport } \\
\text { Science }\end{array} \\
\frac{\text { https://oapub.org/edu/index.php/ejep/article/view/1 }}{908}\end{array}$ & 10 \\
\hline
\end{tabular}

\section{CONTENIDO}

\begin{tabular}{|c|c|c|c|c|c|}
\hline Tipo y diseño & $\begin{array}{c}\text { Población y } \\
\text { Muestra }\end{array}$ & Instrumentos & Aspectos éticos & Resultados & Conclusión \\
\hline $\begin{array}{l}\text { Básica, no } \\
\text { experimental } \\
\text { revisión } \\
\text { bibliográfica }\end{array}$ & $\begin{array}{l}35 \text { miembros del } \\
\text { personal. }\end{array}$ & Entrevista & $\begin{array}{l}\text { Presentación de } \\
\text { información } \\
\text { objetiva }\end{array}$ & $\begin{array}{l}\text { La ponderación de la real situación de la gestión } \\
\text { deportiva en VNU-HCM mostró que algunos } \\
\text { aspectos deben ser ajustados y mejorados, tales } \\
\text { como: los líderes de VNU-HCM, así como los } \\
\text { líderes de las escuelas, no han prestado mucha } \\
\text { atención a la selección y formación de alta calidad. } \\
\text { recursos humanos; la inversión en equipamiento e } \\
\text { infraestructura para el deporte y la actividad física } \\
\text { también es desigual y desenfocada. Hay pocas }\end{array}$ & $\begin{array}{l}\text { La gestión es una contribución } \\
\text { importante y significativa al } \\
\text { desarrollo deportivo de VNU- } \\
\text { HCM en particular, así como } \\
\text { las escuelas en el país en } \\
\text { general. Lo que ayuda al } \\
\text { deporte y al entrenamiento } \\
\text { físico en VNU-HCM para } \\
\text { desarrollarse. }\end{array}$ \\
\hline
\end{tabular}

escuelas que inviertan muy poco en educación

física

Ciencia Latina Revista Científica Multidisciplinar, Ciudad de México, México.

ISN 2707-2207 / ISSN 2707-2215 (en línea), julio-agosto, 2021, Volumen 5, Número 4. https://doi.org/10.37811/cl_rcm.v5i4.789 p.6648 


\section{DATOS}

$\begin{array}{llll}\text { 2. Autor Nono } & \text { Nombre } & \text { Revista }\end{array}$

\begin{tabular}{|c|c|c|c|}
\hline $\begin{array}{l}\text { Shariati, Medi } \\
\text { Rhimi, Meysam } \\
\text { Dastgerdi, Medi }\end{array}$ & (2019) & $\begin{array}{l}\text { El papel de los deportes en el } \\
\text { desarrollo de las relaciones } \\
\text { exteriores de las economías } \\
\text { emergentes (BRIC) }\end{array}$ & $\begin{array}{c}\text { Investigación en gestión deportiva } \\
\text { https://qrsm.atu.ac.ir/article } 11100 . \text { html?lang=en }\end{array}$ \\
\hline
\end{tabular}

\section{CONTENIDO}

\begin{tabular}{|c|c|c|c|c|c|}
\hline Tipo y diseño & $\begin{array}{c}\text { Población y } \\
\text { Muestra }\end{array}$ & Instrumentos & Aspectos éticos & Resultados & Conclusión \\
\hline $\begin{array}{l}\text { Básica, no } \\
\text { experimental } \\
\text { revisión } \\
\text { bibliográfica }\end{array}$ & 50 artículos & $\begin{array}{l}\text { Análisis } \\
\text { documentario }\end{array}$ & $\begin{array}{l}\text { Presentación de } \\
\text { información } \\
\text { objetiva }\end{array}$ & $\begin{array}{l}\text { Las economías emergentes (Brasil, Rusia, India y } \\
\text { China) buscan alcanzar sus metas políticas a nivel } \\
\text { macro en el deporte presentando un programa } \\
\text { integral para el uso de atletas, entrenadores, } \\
\text { árbitros e incluso estudiantes universitarios } \\
\text { deportivos. }\end{array}$ & $\begin{array}{l}\text { los responsables de la toma de } \\
\text { decisiones en la República } \\
\text { Islámica de Irán consideran el } \\
\text { deporte simplemente como un } \\
\text { fenómeno de entretenimiento y } \\
\text { diversión. }\end{array}$ \\
\hline
\end{tabular}

Ciencia Latina Revista Científica Multidisciplinar, Ciudad de México, México. 


\section{DISCUSIÓN}

Sobre la gestión integral, relacionado a los activos fijos, García, Narváez y Álvarez (2019) sustenta cuatro pilares planeamiento, ejecución, control y realización. Este esquema de administración minucioso se articula con los ciclos contables y gerenciales de una manera viable que permite explotar los activos de una asociación para lograr su mayor exhibición de manera confiable y económica. Los autores expresaron que la propuesta del esquema de administración esencial de recursos fijos establece una exitosa herramienta para que la relación tenga la opción de controlar productivamente el periodo de vida y la devaluación de los recursos fijos que reclama; ocuparse de su utilización adecuada asignando obligaciones en cuanto a su seguro y tutela, y controlando los ciclos organizados para su legítima ejecución, prefiriendo la coherencia con el giro previsto y los límites de los beneficios. Complementando lo mencionado por los creadores, se puede ver claramente la importancia de una propuesta de administración exhaustiva para la utilización de los activos de las organizaciones, para esta situación, de acuerdo con una perspectiva contable.

Por otra parte, en el estudio de Kozlov, Budarnikov, Zhuravleva, Gonsales y Lebdeva (2018) en un contexto más deportivo mencionan que la gestión integral para el desarrollo del mercado de los servicios deportivos es crucial para el desarrollo de la nación y su potencial humano. Asimismo, la gestión debe ser sistemático en todas las áreas claves. Para ello, un actor elemental y determinante para que esto sea real es la participación del gobierno, ayudando a crear las condiciones necesarias que faciliten el progreso industrial de los servicios deportivos de un país. En otro contexto, Anaya (2017) quien estudio la gestión integral para la sostenibilidad de las estructuras de salud, hace referencia a que la administración integral avanza formas de vida sólidas entre todos los especialistas hacia la sustentabilidad; Asimismo, averigua cómo trabajar las condiciones de funcionamiento y cuidar el clima con calidad y eficiencia; Precisa que utilizar la administración fundamental fijada en los sujetos como una cultura autoritaria con deferencia por los derechos, valorar la mejoría e inversión de la capacidad humana y extender los estándares de buen trabajo con obligación social hacia sus familias.

Sobre el desarrollo deportivo, Para Gawrtz y Spence (2020) el propósito de una organización deportiva es difícil de conceptualizarla, tanto así que intelectuales y líderes de organizaciones deportivas no han llegado a un término final que les permita 
establecer un propósito consensual, llegando entonces a ser ambiguos sus definiciones. Por otro lado, manifiestan que los investigadores de gestión determinaron mediante evidencias de que el propósito superior de una organización beneficia profundamente al desempeño organizacional. Profundizando la idea de los citados autores, es esencial para el progreso societario que todos estén conectados mediante formas organizativas. En ese sentido, Reyes (2016) especifica que todo movimiento humano es actividad social, y que la cultura se trabaja por códigos, dialectos, articulaciones, indicaciones, estructuras autoritativas, carácter, actitud, en sí misma, es dinámica, de igual manera, declara que igualmente es indefenso y manipulable por diferentes puntos de vista que influyen en la cultura humana y los vínculos sociales. En este sentido, el creador expresa que la cultura deportiva es la consecuencia de un arreglo excepcionalmente desconcertante de las relaciones sociales de creación que suceden en función al juego en varios niveles, contribuyendo adicionalmente a que el juego se concentre en las ciencias sociales, la teoría, la biomecánica, el entrenamiento, la historia, etc. Asimismo, la autora citó a García (1990) quien mencionó que la actividad deportiva comprende diferentes expresiones y en distintos ámbitos sociales. Complementado lo que manifestó Reyes, está bien que el deporte se vea como un organismo dinámico, pues permite que el desarrollo lo sea también no solo afectando a una zona en específica sino descentralizando el desarrollo hacia más sectores. En ese sentido, se complementa, que el desarrollo deportivo es una forma implícita de generar humanos competitivos, sobresalientes e íntegros, asimismo, menciona que, para asegurar la implementación del deporte, el desarrollo requiere ser sostenible, porque hoy en día cada vez el papel del deporte es más importante y estratégico que trae consigo muchos beneficios; entre ellos la formación de carácter y la personalidad de la nación, todo ello, de manera implícita desarrolla y mejora los recursos de una manera sostenible.

En otro contexto, para Di Palma, Tafuri, AScione y Raiola (2018) el crecimiento de los fenómenos relacionados al deporte en sin lugar a dudas uno de los procesos innovadores de los últimos tiempos. Esto se debe a que el desarrollo deportivo se relaciona principalmente con los aspectos educativos, culturales y sociales, asimismo está relacionado con la economía. Es por eso que el deporte es una red multifacética de actividades, experiencias; con organismos detrás de ellas, pues el deporte involucra y afecta también a las organizaciones locales, nacionales e internacionales. Sin embargo, 
los citados autores mencionan que el deporte es visto como entretenimiento y con la capacidad para atraer a una amplia cantidad de espectadores, es por eso, que el deporte aporta mucho a la sociedad.

Sobre la gestión integral y el desarrollo deportivo. Al respecto, Thanh y Viet (2018) mencionó que la gobernanza del deporte tiene un rol fundamental en gran parte de las estructuras de la sociedad, y para las instituciones educativas esta se vuelve crucial para su desarrollo. Sin embargo, menciona el autor que existe desinterés para algunas personas e instituciones, que ocasiona poco interés de los líderes mundiales. Para Thathy Viet, la buena gobernanza en el deporte ayuda a las personas a tener un impacto positivo en su vida, mediante el entrenamiento físico y las actividades deportivas. Ahora, para economías grandes como vienen a ser Rusia, Brasil, China e India, Shariati, Rhimi y Dastgerdi (2019) menciona que estos países buscan alcanzar sus metas políticas y públicas mediante la inclusión e integración del deporte. En ese sentido, presentan un programa mediante la gestión integral de todos los recursos y actores que intervienen para que todos sean beneficiados. En contraste a lo que pasa con los países con economías emergentes, existen muchos países de todas partes del mundo que no lo consideran al deporte como un factor de desarrollo, por eso no invierte mucho en eso y se centran en otros aspectos que para ellos lo consideran importantes, tal es la situación del Estado Iraní, que, según los autores, consideran el deporte simplemente como un fenómeno de entrenamiento y diversión.

En síntesis, la gestión integral es importante para varios propósitos y tipos de desarrollo, además, queda demostrado que depende de las decisiones y prioridades que los países determinen hacia el deporte. Sin embargo, los países con economías emergentes integran al deporte como un mecanismo clave para el desarrollo de su país.

\section{CONCLUSIONES Y RECOMENDACIONES}

En relación a la gestión integral, mediante las investigaciones realizadas queda demostrado que es un componente fundamental para el máximo aprovechamiento las capacidades y recursos institucionales en pro del desarrollo y crecimiento empresarial e institucional, percibir de igual manera su deterioro, de la misma forma en el momento en que ha terminado su periodo de vida para sanear los recursos productivos con los que efectivamente cuentan las instituciones, de esta manera se pueda aplicar otras medidas, sin dejar de lado la gestión integral. 
En cuanto al desarrollo deportivo, en base a los investigaciones analizadas y estudiadas, se concluye que, es una abstracción para crear personas competitivas, íntegras y sobresalientes, porque los deportes se pueden hacer como ejercicios, educación, entretenimiento, recreación, logro, profesión, política, negocios, industria y varios otros aspectos de cultura humana. También, se demostró cómo a través de un proceso evolutivo en el tiempo, el deporte ha llegado a afectar positivamente a otros contextos, incluyendo ciertamente la educación y la escuela, la industria del turismo y lo concerniente cuestiones socioeconómicas.

En relación a la gestión integral y el desarrollo deportivo, se concluye que, existe una clara necesidad de la gestión integral en el desarrollo deportivo internacional, nacional y local; y que el gobierno. Debido a que, la gestión integral es un elemento clave para fortalecer y mejorar diversos procesos, y uno de ellos es el deporte.

En ese sentido, buscando llegar al desarrollo deportivo, se recomienda enfatizar la necesidad de administrar el sistema deportivo desde una perspectiva gerencial a través de procesos operativos y de toma de decisiones basados en criterios económicos de eficiencia y eficacia que puede asegurar resultados satisfactorios en términos de costo.

Además, es importante consolidar medidas de mejora dependientes de una gestión exhaustiva con efectos cuantificables sobre las secuelas de valor, eficiencia, bienestar y seguridad en el trabajo, cuidado del clima y la forma de vida de aseguramiento común. Para ello, es vital que las asociaciones cuenten con opciones de modelos completos que les permitan alcanzar sus metas y satisfacer su objetivo principal de la manera más idónea, aumentando las ventajas y disminuyendo los daños y gastos.

\section{REFERENCIAS}

Alcántara, I., Garza, M., López, A., Magaña, V., Oropeza, O., Puente, S., Rodríguez, D., Lucatello, S., Ruiz, N., Tena, R., Urzúa, M. y Vázquez, G. (2019). Gestión integral de riesgo de desastres en México: reflexiones, retos y propuestas de transformación de la política pública desde la academia. Investigaciones Geográficas, 98. https://doi.org/10.14350/rig.59784

Anaya, A. (2017). Modelo de salud y seguridad en el trabajo con gestión integral para la sustentabilidad de las organizaciones (SSeTGIS). Ciencia \& Trabajo, 19(59), 95-104. https://doi.org/10.4067/s0718-24492017000200095

Arias, E., Rieble, S., Álvarez, H., Rivera, M., Viteri, A., López, Á., Pérez, M., Vásquez, 
M., Bergamaschi, A., Noli, A., Ortiz, M. y Scannone, R. (2020). La educación en tiempos del coronavirus: Los sistemas educativos de América Latina y el Caribe ante COVID-19. Banco Interamericano de Desarrollo. http://dx.doi.org/10.18235/0002337

Arronte, J. y Ferro, B. (2020). El proceso de iniciación deportiva escolar en voleibol: una mirada de renovación para su aprendizaje. Podium. Revista de Ciencia y Tecnología En La Cultura Física, 15(1), 127-141.

Babiak, K., Thibault, L. y Willem, A. (2017). Investigación cartográfica sobre las relaciones interorganizacionales en la gestión del deporte: panorama actual y perspectivas de investigación futura. Journal of Sport Management, 32(3), 272294. https://doi.org/https://doi.org/10.1123/jsm.2017-0099

Di Palma, D., Tafuri, D., AScione, A. y Raiola, G. (2018). Desarrollo social, turístico y educativo a través del deporte. Journal of Physical Education and Sport, 18(1), 473-478. https://doi.org/10.7752/jpes.2018.s167

Dowling, M., Leopkey, B. y Smith, L. (2018). Governance in Sport: A Scoping Review. Human Kinetics Journals, 32(5), 438-451. https://doi.org/https://doi.org/10.1123/jsm.2018-0032

García, L., Narváez, C. y Erazo, J. (2019). Gestión integral de activos fijos con enfoque en el control contable administrativo. Visionario Digital, 3(2), 305-328. https://doi.org/10.33262/visionariodigital.v3i2.479

Gwartz, E. y Spence, K. (2020). Capitalismo consciente y deporte: Explorando un propósito superior en una organización deportiva profesional. Sport Management Review, 23(4). https://doi.org/https://doi.org/10.1016/j.smr.2019.09.002

Kozlov, A., Budarnikov, A., Zhuravleva, Y., Gonsales, S. y Lebdeva, O. (2018). Desarrollo del mercado de servicios deportivos en las condiciones actuales. The journal of social sciences researcH, 53, 144-148. https://www.elibrary.ru/item.asp?id=36544846

Leiton, N. y Revelo, W. (2017). Gestión integral de residuos sólidos en la empresa CYRGO SAS. Tendencias, 18(2), 103. https://doi.org/10.22267/rtend.171802.79

Lizarzaburu, E., Barriga, G., Noriega, E. y Burneo, K. (2018). Gestión integral de riesgos y antisoborno: un enfoque operacional desde la perspectiva ISO $31000 \mathrm{E}$ 
ISO 37001. Universidad \& Empresa, 21(36), 79. https://doi.org/10.12804/revistas.urosario.edu.co/empresa/a.6089

Maqueira, G. Brito, E., Recalde, A., Arévalo, J., Sáenz, J. y Sandoval, M. (2017). Tendencias y manifestaciones del temperamento. Relación con el desempeño social y físico-deportivo en estudiantes. Revista Cubana de Investigaciones Biomedicas, 36(2), 127-137.

Ministerio de Salud (2006). Modelo de Abordaje de promoción de la salud en el Perú. Dirección General de Promoción de Salud. https://crolimacallao.org.pe/documentos/normas/MODELO DE ABORDAJE PROMOCION DE LA SALUD PERU.pdf

Mujika, I., Halson, S., Burke, L. M., Balagué, G. y Farrow, D. (2018). Un enfoque multifactorial e integrado de la periodización para un rendimiento óptimo en deportes individuales y de equipo. Human Kinetics Journals, 3(5), 538-561. https://doi.org/https://doi.org/10.1123/ijspp.2018-0093

Parent, M. y Hoye, R. (2018). El impacto de los principios de gobernanza en las prácticas y el rendimiento de las organizaciones deportivas: una revisión sistemática. Cogent Social Sciences, https://doi.org/https://doi.org/10.1080/23311886.2018.1503578

Parra, E. y Salazar, A. (2017). La gestión integral del agua en dos consejos de cuenca del noroeste de México. Entreciencias: Diálogos En La Sociedad Del Conocimiento, $5(15)$ 79-94. https://doi.org/10.22201/enesl.20078064e.2017.15.62580

Peachey, J. W., Cohen, A., Shin, N. y Fusaro, B. (2018). Desafíos y estrategias para construir y mantener asociaciones interinstitucionales en el deporte para el desarrollo y la paz. Revisión de La Gestión Deportiva, 21(2), 160-175. https://doi.org/https://doi.org/10.1016/j.smr.2017.06.002

Pérez, N. (2007). La importancia de la promoción a la salud dentro de las escuelas de educación básica. Universidad Autónoma del Estado de Hidalgo. http://dgsa.uaeh.edu.mx:8080/bibliotecadigital/bitstream/handle/231104/457/La; jsessionid=7D43AF909AAC541652C150B083473C49? sequence $=1$

Prato, L., Alcaraz, S., Ramis, Y. y Torregrossa, M. (2020). Experiencias del entorno deportivo de origen al asesorar a esgrimistas migrantes. Pensamiento 
Psicológico, 18(02). https://doi.org/10.11144/Javerianacali.PPSI18-2.eedo

Rabwan, K. A. (2020). Análisis del Desarrollo Deportivo en la Regencia Bima desde la Perspectiva Epistemológica. Advances in Social Science, Education and Humanities Research, 443. https://doi.org/https://dx.doi.org/10.2991/assehr.k.200620.057

Reátegui, E. (2008). Saber sobre promoción de la salud en familias y personal de salud. Universidad Nacional de Trujillo. https://dspace.unitru.edu.pe/handle/UNITRU/5411

Reyes, J. (2020). Omnivorismo deportivo: Estratificación social de la práctica deportiva en Chile. Revista Internacional de Sociología del Deporte. https://doi.org/https://doi.org/10.1177\%2F1012690220959102

Reyes, L. (2016). Gerencia Deportiva para la Masificación Deportiva Planificada y Ejecutada por las Organizaciones Sociales. Críticas a Modelos, Experiencias y Propuesta de Acompañamiento Metodológico. Revista Scientific, 1(1), 231-248. https://doi.org/http://doi.org/10.29394/scientific.issn.25422987.2016.1.1.13.231-248

Sadat, Z. y Shajie, K. (2017). Diseño y desarrollo del plan estratégico del deporte universitario (estudio de caso: Oficina de Educación Física de la Universidad ferdowsi). Sport Scinces Research Institute of Iran, 5(12), 61-78. https://doi.org/https://dx.doi.org/10.22089/res.2017.939

Shariati, M., Rhimi, M. y Dastgerdi, M. (2019). El papel de los deportes en el desarrollo de las relaciones exteriores de las economías emergentes (BRIC). Investigación en gestión $\quad$ deportiva, $\quad$ 63-109. https://doi.org/https://dx.doi.org/10.22054/qrsm.2019.11100

Sarria, A. y Del Villar, F. (2014). Promoción de la salud en la comunidad (Universidad Nacional de Educación a Distancia - UNED (ed.)). https://dialnet.unirioja.es/servlet/libro?codigo=565425

Thanh, P. y Viet, T. (2018). La gestión de los recursos deportivos en Hochiminh Universidad Nacional, Vietnam. European Journal of Physical Educatión and Sport Science, 4(10). https://doi.org/http://dx.doi.org/10.46827/ejpe.v0i0.1908 